\title{
Early Age Strength Healing Effect of Cementitious Composite Incorporated Self- Healing Microcapsule
}

\author{
Xingang WANG ${ }^{1 *}$, Xingjing WANG ${ }^{1}$, Yuhao $\mathrm{XIE}^{2}$, Wei $\mathrm{XU}^{1}$ \\ ${ }^{1}$ School of Civil Engineering and Architecture, Nanchang University, Nanchang 330031, China \\ ${ }^{2}$ China West Construction Group Co., Ltd., Chendu 610015, China \\ cross ref http://dx.doi.org/10.5755/j02.ms.22841
}

Received 27 February 2019; accepted 02 September 2019

\begin{abstract}
This paper aims to explore early age strength healing effect of cementitious composite incorporated self-healing microcapsule and curing activity of self-healing microcapsule. Particle characteristic of the microcapsule and micromorphology of cement paste incorporated the microcapsule were characterized by SEM. Curing activity of the microcapsule was analyzed by macroscopic solidification test, DSC and TGA. The influence factors of early age strength healing ratio in cementitious composite incorporated self-healing microcapsule were studied. The results showed that epoxy resin microcapsule had favorable micromorphology. Epoxy resin microcapsule core material had good curing activity and possessed the ability to play the healing role in cementitious composite. Healing temperature and healing age had less impact on the healing effect of the microcapsule in cementitious composite. The microcapsule could be kept good shape in cement paste and combined with cement paste closely. The microcapsule also could rupture and had better dispersion in cement paste. The main fracture behavior of the microcapsule was based on small hole ruptured when cement paste did not occur macro damage. When pre-loading was $0.75 \sigma_{\max }$, the particle size of microcapsules was range from $75 \mu \mathrm{m}$ to $150 \mu \mathrm{m}$, proportion of epoxy curing agent was $20 \%$ and proportion of the microcapsule was $6 \%$, early age strength healing ratio reached the highest of $24.1 \%$.
\end{abstract}

Key words: microcapsule, healing, early age, strength, cementitious composite.

\section{INTRODUCTION}

Self-healing microcapsules with microencapsulated healing agents have been developed in polymers and composites [1-3]. To introduce self-healing microcapsules into cementitious composites provided the inspiration and the fresh perspective for solving the important problem of the crack and its healing in cementitious composites. Since then, cementitious composites incorporated self-healing microcapsules have become one of the research hotspots $[4,5]$.

Yang [6] showed that the addition of microencapsulated methylmethacrylate based healing agent into carbon microfiber-reinforced mortar can greatly improve the crack resistance and toughness under fatigue loading. $\mathrm{Hu}$ [7] incorporated UF/E microcapsules into cement paste, and the healing capability of microcapsules was verified by recovery of compressive strength of set cement after pre-loading. Dong [8] researched the influence of the dosage of Urea-formaldehyde resin/epoxy resin (UF/E) microcapsules on self-healing efficiency, strength, and permeability of cement-based materials. But what he had ignored was the hydrated reaction in the early ages of cement-based materials could endow the composites with some compressive strength. Li [9] added Melamine Urea-Formaldehyde (MUF/E) microcapsules into cement paste, and the crack-healing results was confirmed by SEM/EDS. Dong [10] introduced theoretical background of curing reaction mechanism, the healing

\footnotetext{
* Corresponding author. Tel.: +86-0791-83969655; fax: +86-0791-83969655. E-mail address: wangxingang@ncu.edu.cn (X. Wang)
}

effects of UF/E microcapsules in cementitious material was evaluated by crack-healing ratio, healing ratio of mechanical property. To the knowledge of the authors, cementitious composites embedded with self-healing microcapsules were focused on healing effects of mechanical properties, especially long age strength healing $[11,12]$. But there was lack of the research on early age strength healing effect of cementitious composites incorporated self-healing microcapsules and curing activity of self-healing microcapsules.

The objective of the present investigation explored early age strength healing effect of cementitious composite incorporated self-healing microcapsules and curing activity of self-healing microcapsules. Epoxy resin microcapsules were prepared by in situ polymerization method. Particle characteristic of the microcapsule and micromorphology of cement paste incorporated the microcapsule were characterized by SEM. Curing activity of the microcapsule was analyzed by macroscopic solidification test, DSC and TGA. Calculation formula of early age strength healing ratio of cementitious composite was improved. The influence factors of early age strength healing ratio in cementitious composite incorporated self-healing microcapsule was studied, including pre-loading, particle size range of microcapsule, proportion of epoxy curing agent and proportion of the microcapsule.

\section{EXPERIMENTS}

\subsection{Materials}

Industrial grade epoxy resin E-51 was purchased from Hui Xin chemical co. Ltd. Guangzhou, China. Urea, 
formaldehyde aqueous solution (37 wt.\%), triethanolamine, SDBS (sodiumdodecyl benzene sulfonate), ammonium chloride, sodium hydroxide, resorcinol and epoxy curing agent (DMP-30) were provided from Sinopharm Chemical Reagent Co. Ltd. China. Laboratory tap water was used for all experiments. All chemicals were of analytical grade and were used without further purification.

An ordinary Portland cement (CEMI 42.5 N) was supplied by Ya Dong cement co. Ltd. Nanchang, China. Chinese ISO standard sand purchased from $\mathrm{Ai} \mathrm{Si} O$ standard sand co. Ltd. Xiamen, China. High performance polycarboxylic water reducer (standard type) obtained from Nanchang technology innovation building materials co. Ltd., China. The solid content is $20 \%$ and the water reducing rate is more than $25 \%$.

\subsection{Preparation and characterization of the microcapsule}

The UF/E microcapsules were prepared by in-situ polymerization as following four steps:

1. Preparation of the UF resin prepolymer. Urea and formaldehyde aqueous solution (37 wt.\%) were mixed in a 1:2 molar ratios [13]. Once all the urea had dissolved, triethanolamine was used to adjust the $\mathrm{pH}$ to $8.0-9.0$. Then, the reaction system was stirred as a rate of $450 \mathrm{rpm}$ for $1 \mathrm{~h}$ at $70^{\circ} \mathrm{C}$ to afford the UF resin prepolymer.

2. Preparation of an oil-in-water emulsion. The epoxy resin E-51 for the core was added to an aqueous solution of SDBS with stirring at a rate of $1300 \mathrm{rpm}$ $40 \mathrm{~min}$ at $50{ }^{\circ} \mathrm{C}$ to form the emulsion.

3. Capsules shell formation stage. UF resin prepolymer was added to the emulsion of core material that the mass ratio of core material and shell material was $1: 1$. The $\mathrm{pH}$ was adjusted to 3.0 using ammonium chloride and sodium hydroxide. Then, the reaction system was stirred at a rate of $900 \mathrm{rpm}$ for $1 \mathrm{~h}$ at $50{ }^{\circ} \mathrm{C}$.

4. Capsules shell solidification stage. Resorcinol was added to reaction system, and the reaction system was stirred at a rate of $900 \mathrm{rpm}$ for $1.5 \mathrm{~h}$ at $60^{\circ} \mathrm{C}$. Finally, the microcapsules were isolated by filtration, drying and sifting.

Particle characteristic of the microcapsule and micromorphology of cement paste incorporated the microcapsule were characterized by Quanta200F environmental scanning electron microscope (SEM) produced by FEI company. Curing activity of the microcapsule was investigated by DSC800 differential scanning calorimeter (DSC) and TGA4000 thermo gravimetric analyzer (TGA) produced by PERKINELMER company.

Fig. 1 presents ESEM images of the microcapsule. Fig. 1 shows that the microcapsule had favorable micromorphology, which was characterized by little deformation and full particle shape, good dispersion, excellent surface texture, and efficient utilization of the shell particles. Particle size range of the microcapsule is mainly distributed in $75-300 \mu \mathrm{m}$.

\subsection{Preparation and test of cementitious composite incorporated the microcapsule}

Preparation of cement mortar incorporated the microcapsule was as following: first, cement, sand and microcapsule were mixed together; then water, water reducer and epoxy curing agent were placed in the mixer; next, the mixture was slowly stirred for $30 \mathrm{~s}$, after which the mixer was paused for $90 \mathrm{~s}$, then stirring was resumed for $60 \mathrm{~s}$.

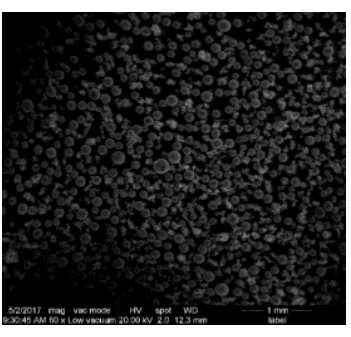

a

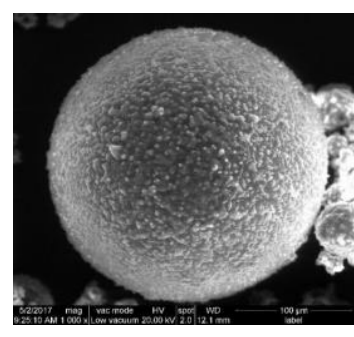

b
Fig. 1. SEM images of the microcapsule with different magnification: $a$-overall; $b$-single

And repeat the process 4-5 times. Prismatic specimens with dimensions of $40 \mathrm{~mm} \times 40 \mathrm{~mm} \times 160 \mathrm{~mm}$ were cast for mechanical behavior tests. The specimens were demolded after $24 \mathrm{~h}$ and maintained in standard curing chamber (temperature $20 \pm 2{ }^{\circ} \mathrm{C}$, relative humidity $>95 \%$ ). Three prismatic specimens of each group were prepared for mechanical behavior tests and then the average value was calculated.

Pre-loading method and compressive strength test was performed in a following way: the maximum compressive stress $\left(\sigma_{\max }\right)$ of specimens was measured by the loading rate of $0.5 \mathrm{KN} / \mathrm{s}$ under the hydraulic machine. Then, the specimens were pre-loaded with $0.6 \sigma_{\max }, 0.75 \sigma_{\max }$ or $0.9 \sigma_{\max }$. Once reach to the preset load, the constant pressure was unloaded after $1 \mathrm{~min}$, and the pre-loaded specimens were maintained in a standard curing chamber. Cement mortar specimens were broken into two parts at 3 days, one was used to measure $\sigma_{\max }$, the other one was pre-loaded under the pressure machine. Then, the specimens were maintained in standard curing chamber until 7 days. Finally, 7 days' compressive strength of the specimens was tested.

\subsection{Mix proportions of cementitious composite incorpo-rated the microcapsule}

According to the previous research, in different incorporated methods of the microcapsule, the method to use the microcapsule instead of sand has the least effect on strength of cement mortar incorporated the microcapsule [14]. In this paper, microcapsules were used to replace the same mass of sand, which was a part cement mortars. Mix proportions of cement mortar incorporated the microcapsule are shown in Table 1. All cement mortars were prepared at the mass ratio $(\mathrm{W} / \mathrm{B})$ of 0.5 . In order to ensure the fluidity of cement mortars is $200 \pm 10 \mathrm{~mm}$, water reducer was used in cement mortars.

Based on the above mix proportions, the influence factors of early age strength healing ratio in cementitious composite incorporated self-healing microcapsule, 
including pre-loading, particle size range of microcapsule, proportion of epoxy curing agent and proportion of the microcapsule, were investigated in this paper. Healing situations of cement mortars incorporated self-healing microcapsule are given in Table 2. Compressive strengths of cement mortars incorporated self-healing microcapsule are given in Table 3 .

Due to continuous cement hydration, certain degree of strength recovery of cement mortars after pre-loading takes place. The influence of strength improvement resulted from cement hydration should be eliminated. Thus, early age strength healing ratio was determined based on Eq. 1.

$$
\eta_{\text {early age }}=\frac{\left\{\left(F_{\text {healed }}-F_{\text {initial }}\right)-\left(f_{\text {healed }}-f_{\text {initial }}\right)\right\}}{f_{\text {healed }}} \times 100 \%
$$

7 days' strength healing ratios of cement mortars incorporated self-healing microcapsule are also given in Table 3 . where $\eta_{\text {early age }}$ is early age strength healing ratio that is based on compressive strength, $F_{\text {healed }}$ is the compressive strength of specimens incorporated microcapsules after pre-loading, healing and maintain, $F_{\text {initial }}$ is the compressive strength of specimens incorporated microcapsules before pre-loading, healing and maintain, $F_{\text {healed }}$ is the compressive strength of specimens that unmixed with microcapsules after pre- loading, healing and maintain and $F_{\text {initial }}$ is the compressive strength of specimens that unmixed with microcapsules before pre-loading, healing and maintain.

\section{RESULTS AND DISCUSSION}

\subsection{Pre-loading}

Fig. 2 corresponds with the experiments $1-3$ in Table 2. Fig. 2 shows that early age strength healing ratio of different pre-loading, when the proportion of epoxy curing agent was $9 \%$, the proportion of microcapsule was $4 \%$ and particle size range of microcapsule was $150-300 \mu \mathrm{m}$.

When the pre-loading was $0.6 \sigma_{\max }-0.9 \sigma_{\max }$, early age strength healing ratio was climb up and then decline. When the pre-loading was $0.75 \sigma_{\max }$, the healing ratio reached highest was $7.5 \%$. In a certain range, the number of microcracks in specimens increased with increased of preloading. There were much more microcapsules cracked, which could heal the microcracks caused by the preloading. Hence, the healing ratio of early age strength increased at the same time. However, when the pre-loading was $0.9 \sigma_{\max }$, the partial specimens already had macroscopic cracks. These macroscopic cracks were too large to be healed with the limited microcapsules.

Table 1. Mix proportions of cement mortar incorporated the microcapsule

\begin{tabular}{|c|c|c|c|c|c|}
\hline Cement, $\mathrm{g}$ & Water, $\mathrm{g}$ & Sand, $\mathrm{g}$ & Microcapsule, $\mathrm{g}$ & Epoxy curing agent, $\mathrm{g}$ & Water reducer, $\mathrm{g}$ \\
\hline 450 & 225 & $1242-1296$ & $54-108$ & $0-21.6$ & $2.25-4.00$ \\
\hline
\end{tabular}

Table 2. Healing situations of cement mortars incorporated self-healing microcapsule

\begin{tabular}{|c|c|c|c|c|}
\hline No. & Pre-loading & Epoxy curing agent/microcapsule & Microcapsule /(microcapsule + sand) & Particle size range of microcapsule \\
\hline 1 & $0.6 \sigma_{\max }$ & $9 \%$ & $4 \%$ & $150-300 \mu \mathrm{m}$ \\
\hline 2 & $0.75 \sigma_{\max }$ & $9 \%$ & $4 \%$ & $150-300 \mu \mathrm{m}$ \\
\hline 3 & $0.9 \sigma_{\max }$ & $9 \%$ & $4 \%$ & $150-300 \mu \mathrm{m}$ \\
\hline 4 & $0.75 \sigma_{\max }$ & $0 \%$ & $4 \%$ & $75-150 \mu \mathrm{m}$ \\
\hline 5 & $0.75 \sigma_{\max }$ & $9 \%$ & $4 \%$ & $75-150 \mu \mathrm{m}$ \\
\hline 6 & $0.75 \sigma_{\max }$ & $20 \%$ & $4 \%$ & $75-150 \mu \mathrm{m}$ \\
\hline 7 & $0.75 \sigma_{\max }$ & $30 \%$ & $4 \%$ & $75-150 \mu \mathrm{m}$ \\
\hline 8 & $0.75 \sigma_{\max }$ & $40 \%$ & $4 \%$ & $75-150 \mu \mathrm{m}$ \\
\hline 9 & $0.75 \sigma_{\max }$ & $20 \%$ & $4 \%$ & $750-300 \mu \mathrm{m}$ \\
\hline 10 & $0.75 \sigma_{\max }$ & $20 \%$ & $5 \%$ & $75-150 \mu \mathrm{m}$ \\
\hline 11 & $0.75 \sigma_{\max }$ & $20 \%$ & $6 \%$ & $75-150 \mu \mathrm{m}$ \\
\hline 12 & $0.75 \sigma_{\max }$ & $20 \%$ & $7 \%$ & $75-150 \mu \mathrm{m}$ \\
\hline 13 & $0.75 \sigma_{\max }$ & $20 \%$ & $8 \%$ & $75-150 \mu \mathrm{m}$ \\
\hline
\end{tabular}

Table 3. Compressive strengths and early age strength healing ratios of cement mortars incorporated self-healing microcapsule

\begin{tabular}{|c|c|c|c|c|c|}
\hline No. & $F_{\text {healed }}, \mathrm{MPa}$ & $F_{\text {initial }}, \mathrm{MPa}$ & $f_{\text {healed, }} \mathrm{MPa}$ & $f_{\text {initial }}, \mathrm{MPa}$ & 7 days strength healing ratio, $\%$ \\
\hline 1 & 32.20 & 27.62 & 23.41 & 20.11 & 5.5 \\
\hline 2 & 32.31 & 28.04 & 20.86 & 18.16 & 7.5 \\
\hline 3 & 30.55 & 25.39 & 22.80 & 19.05 & 6.2 \\
\hline 4 & 10.71 & 9.71 & 13.18 & 12.34 & 1.2 \\
\hline 5 & 24.27 & 21.98 & 16.66 & 15.72 & 8.1 \\
\hline 6 & 25.31 & 19.14 & 14.39 & 10.22 & 13.9 \\
\hline 7 & 34.81 & 27.96 & 26.21 & 21.98 & 10.0 \\
\hline 8 & 28.27 & 24.63 & 17.89 & 15.52 & 7.1 \\
\hline 9 & 32.51 & 26.75 & 18.70 & 15.27 & 12.5 \\
\hline 10 & 36.07 & 27.66 & 20.59 & 15.33 & 15.3 \\
\hline 11 & 48.08 & 41.10 & 15.45 & 12.20 & 24.1 \\
\hline 12 & 40.58 & 30.78 & 23.49 & 17.79 & 17.5 \\
\hline 13 & 29.32 & 23.81 & 18.26 & 15.57 & 15.4 \\
\hline
\end{tabular}


As a result, early age strength healing ratio was decreased. Moreover, it could be predicted that when the pre-loading was $\sigma_{\max }$, cement mortar specimens would appear the macro-damage and lose the self-healing ability.

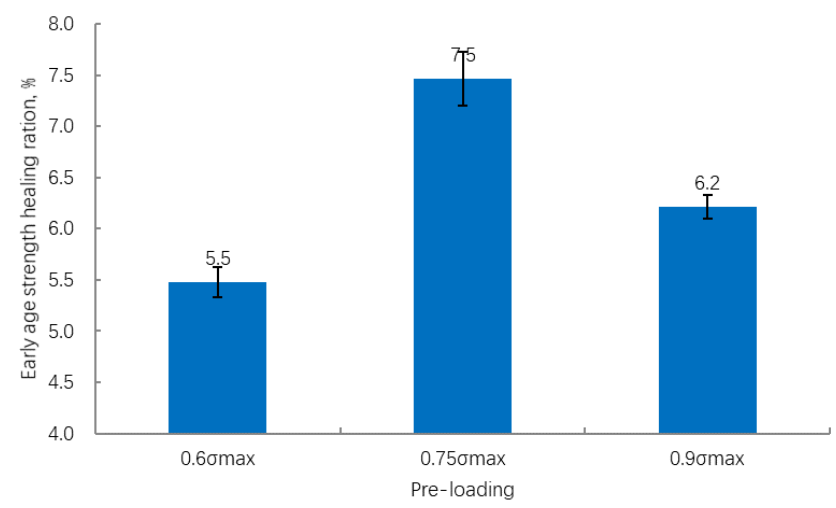

Fig. 2. Early age strength healing ratio of different pre-loading

\subsection{Particle size range of the microcapsule}

Fig. 3 corresponds with the experiments 2, 5, 6 and 9 in presented in Table 2. Fig. 3 shows that early age strength healing ratio of different particle size range of the microcapsule, when the pre-loading was $0.75 \sigma_{\max }$, the proportion of microcapsule was $4 \%$ and the proportion of epoxy curing agent was $9 \%$ and $20 \%$.

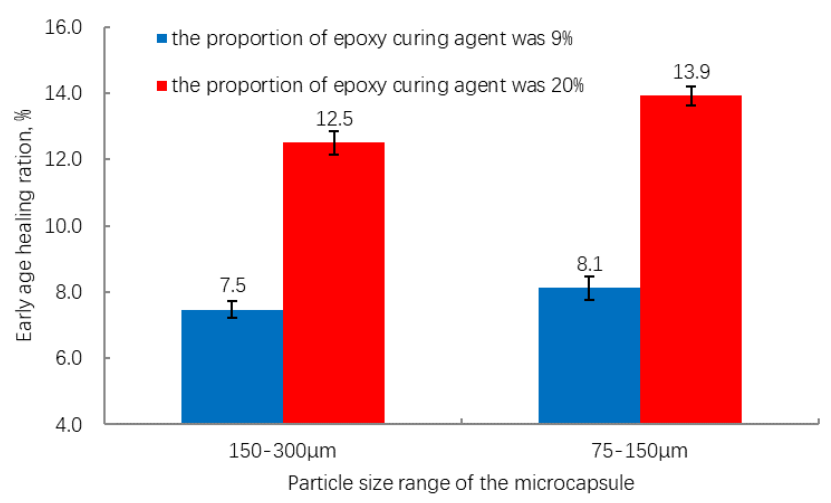

Fig. 3. Early age strength healing ratio of different particle size range of microcapsule

When the proportion of epoxy curing agent was $9 \%$ and $20 \%$, and the particle size of microcapsules was in the range from $75 \mu \mathrm{m}$ to $150 \mu \mathrm{m}$, early age strength healing ratio was in the $8.1 \%$ and $13.9 \%$, respectively. When the particle size of microcapsules was range from $75 \mu \mathrm{m}$ to $150 \mu \mathrm{m}$, early age strength healing ratio was higher than that was $150-300 \mu \mathrm{m}$. The reason may be that smaller microcapsules were easier to rupture and play the healing role when the cracks generated [15]. Therefore, in the appropriate range, particle size of microcapsule was smaller and particle size range of microcapsule was narrower the better for improvement of early age strength healing ratio was obtained.

\subsection{Proportion of epoxy curing agent}

Fig. 4 corresponds with the experiments 4-8 presented in Table 2. Fig. 4 shows that early age strength healing ratio of different proportion of epoxy curing agent, when the pre-loading was $0.75 \sigma_{\max }$, the proportion of microcapsule was $4 \%$ and particle size range of the microcapsule was $150-300 \mu \mathrm{m}$.

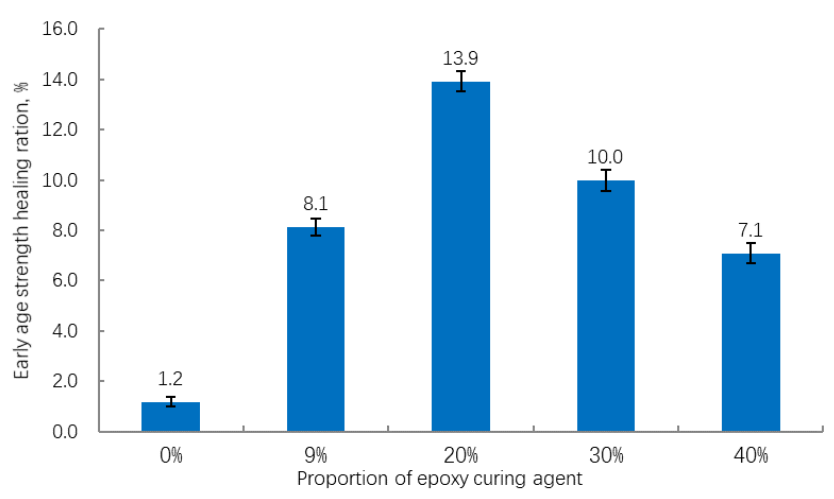

Fig. 4. Early age strength healing ratio of different proportion of epoxy curing agent

When the proportion of epoxy curing agent was $0-40 \%$, early age strength healing ratio was climb up and then decline. When the proportion of epoxy curing agent was $20 \%$, the healing ratio reached highest was $13.9 \%$. And when the proportion of epoxy curing agent was $0 \%$, the healing ratio reached lowest was $1.2 \%$. The curing reaction of core material and curing agent could not happen without the participation of curing agent.

\subsection{Proportion of the microcapsule}

Fig. 5 corresponds with the experiments 6 and 10-13 presented in Table 2. Fig. 5 shows that early age strength healing ratio of different proportion of microcapsule, when the pre-loading was $0.75 \sigma_{\max }$, the proportion of epoxy curing agent was $20 \%$ and particle size of microcapsules was in the range from $75 \mu \mathrm{m}$ to $150 \mu \mathrm{m}$.

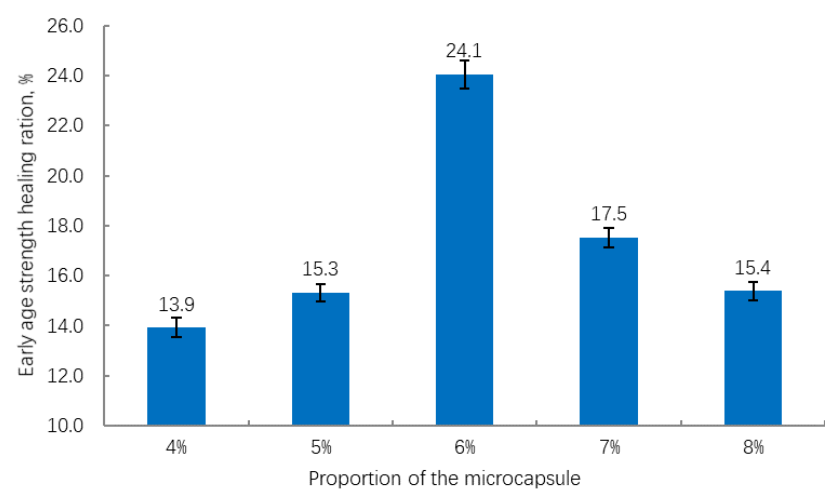

Fig. 5. Early age strength healing ratio of different proportion of the microcapsule

When the proportion of microcapsule was 4-8\%, early age strength healing ratio was climb up and then decline. When the proportion of microcapsule was $6 \%$, the healing ratio reached highest was $24.1 \%$. The microcapsules content could somehow influence the structure of specimens. When the content of microcapsules was below $6 \%$, the quantity of healing agent could cure the microcrack generated. But when the content of microcapsules was over $6 \%$, maybe the structure of specimens changed a lot, and the number of cracks increased a lot. The size of microcracks increased a lot too. 


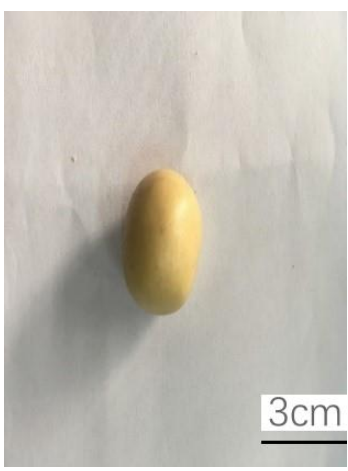

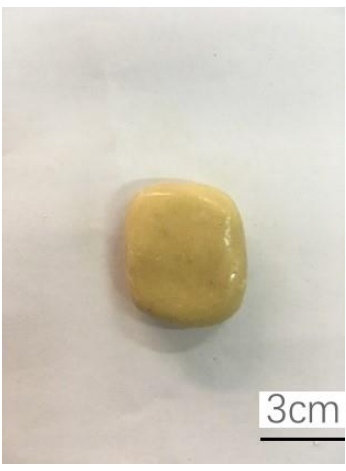

$\mathrm{b}$

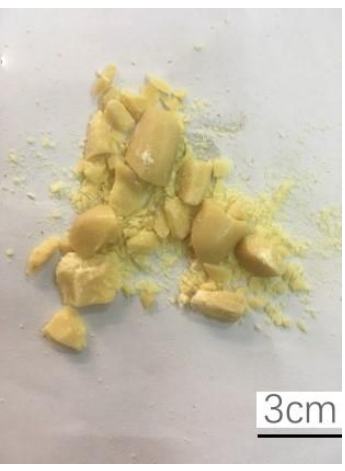

c

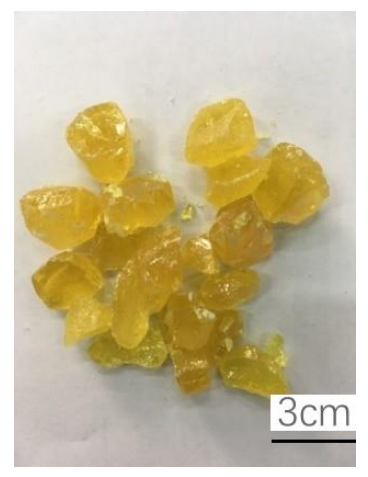

d

Fig. 6. Core material curing effect diagram: $a$-grinded the microcapsule powder and mixed with epoxy curing agent; $b$-the spherical mixture in a had been shelved for 90 min; $\mathrm{c}$-the spherical mixture in $\mathrm{b}$ had been smashed; $\mathrm{d}$-cured epoxy resin had been smashed

The limited microcapsule was not able to cure the microcracks completely. Thus, $6 \%$ was optimal proportion of the microcapsule.

\subsection{Curing activity of the microcapsule}

Fig. 6 shows that curing activity of the microcapsule core material was proved by macroscopic solidification test. Fig. 6 a shows that spherical mixture acquired by grinded the microcapsule powder and mixed with epoxy curing agent at room temperature. The spherical mixture in Fig. 6 a was very soft and could be knead in various shapes. Fig. $6 \mathrm{~b}$ shows that the spherical mixture had been shelved for $90 \mathrm{~min}$ at room temperature. It was found that the spherical mixture hardened and the heat released. It indicated that temperature and time have less impact on the curing reaction. Namely, the microcapsule performed well at room temperature. Hence, it could work in a wide temperature range of environments $[16,17]$. Fig. $6 \mathrm{c}$ shows that spherical mixture had been smashed by a hammer after 1 day. It can be concluded that the spherical mixture had a high intensity when the powder of microcapsules was mixed with epoxy curing agent and reacted for one day. Fig. $6 \mathrm{~d}$ shows that cured epoxy resin had been smashed by a hammer. The situation was similar to Fig. $6 \mathrm{c}$. The epoxy resin solidified with high hardening strength and accompanied by heat released.

Obviously, microcapsule wall material would not react with the core material (epoxy). So, it can be concluded from Fig. 6 that grinded the microcapsule powder and mix with epoxy curing agent would harden at room temperature, and the harden situation was very similar to the cured epoxy resin. Because microcapsule wall blended with epoxy curing agent did not harden, so core material which flows out from the grinded microcapsule could reacted with epoxy curing agent. Namely the microcapsule core material had good curing activity.

Curing activity of the microcapsule core material could also be analyzed through TGA and DSC curves. Fig. 7 curve a, b, c and $d$ are the TGA curves of microcapsule, microcapsule shell material, epoxy resin and epoxy curing agent respectively. Fig. 8 curve a, b are DSC curves of grinded the microcapsule powder mixed with epoxy curing agent and microcapsule wall blended with epoxy curing agent respectively.
Fig. 7 shows that there are almost no mass loss of microcapsule and epoxy resin before $250{ }^{\circ} \mathrm{C}$, and there are almost no mass loss of epoxy curing agent before $150{ }^{\circ} \mathrm{C}$.

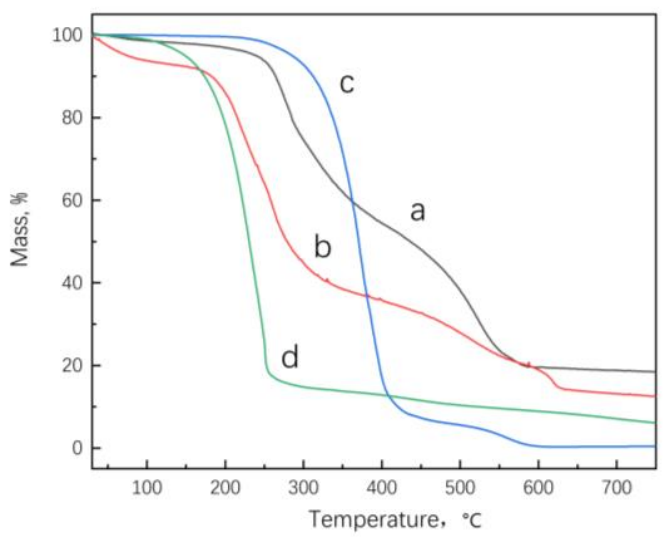

Fig.7. TGA curves: $a-$ microcapsule; $b$-microcapsule shell materials; $\mathrm{c}$-epoxy resin; $\mathrm{d}$-epoxy curing agent

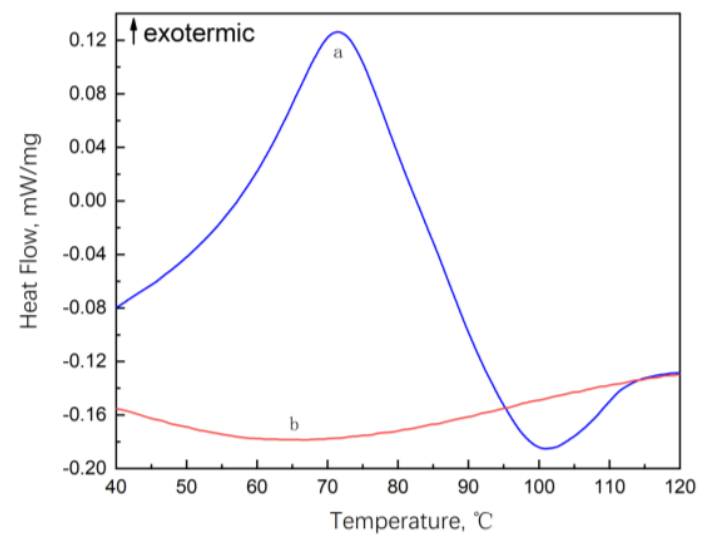

Fig. 8. DSC curves: a-grinded the microcapsule powder mixed with epoxy curing agent; $b$-microcapsule wall blended with epoxy curing agent

But the mass of microcapsule shell material decreases slightly before $100{ }^{\circ} \mathrm{C}$ and fall sharply in about $200{ }^{\circ} \mathrm{C}$.

Combining TGA curves in Fig. 7 , what we can conclude from Fig. 8 was that the exothermic peak of curve a was not caused by the decomposition of microcapsules and epoxy curing agent. Curve b in Fig. 8 
had no obvious exothermic peak and endothermic peak, so the microcapsule wall did not react with epoxy curing agent. Thus, the reason for occurrence of exothermic peak at $72{ }^{\circ} \mathrm{C}$ in the curve a was the heat released from the reaction of the microcapsule core and epoxy curing agent. And the reason for occurrence of endothermic peak at $100{ }^{\circ} \mathrm{C}$ in the curve a was the solidified product of core material absorbs heat decomposition. In conclusion, DSC test further proved that microcapsule core material had good curing activity, so the microcapsule possessed the ability to play the healing role in cementitious composite.

\subsection{Micromorphology of cement paste incorporated the microcapsule}

The small fragments were selected from the crushed cement paste specimens with added microcapsules. Micromorphology of cement paste incorporated the microcapsule was acquired by using SEM observation the small fragments. Fig. 9 a shows that the microcapsules had better dispersion in cement paste section.

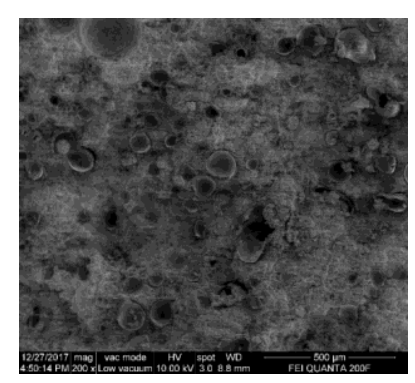

a

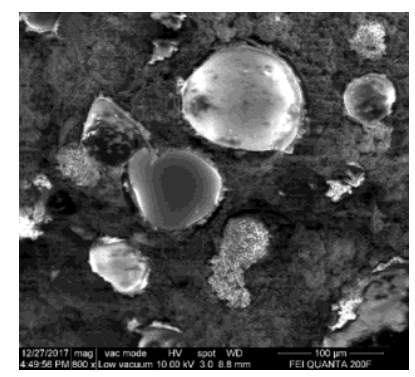

c

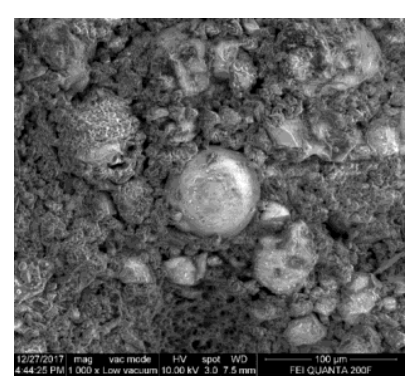

b

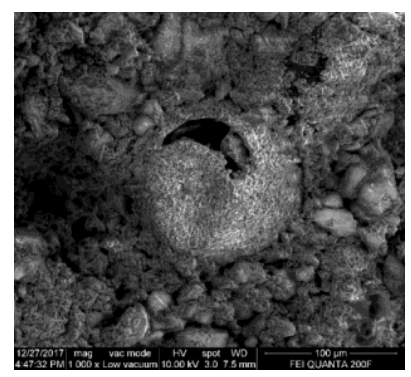

d
Fig. 9. ESEM images of cement paste incorporated the microcapsule

\section{CONCLUSIONS}

1. The method to incorporate the microcapsule to cementitious composite is the microcapsule instead of the mass of sand was proposed. When pre-loading was $0.75 \sigma \mathrm{max}$, the particle size of microcapsules was in the range from $75 \mu \mathrm{m}$ to $150 \mu \mathrm{m}$, proportion of epoxy curing agent was $20 \%$ and proportion of the microcapsule was $6 \%$, early age strength healing ratio reached the highest of $24.1 \%$. The addition of the microcapsule could not only play the healing role but also increased strength of the cement mortar.

2. Epoxy resin microcapsule had favorable micromorphology. Epoxy resin microcapsule core material had good curing activity and possessed the ability to play the healing role in cementitious composite. Temperature and time had less impact on the curing reaction. Namely, healing temperature and healing age had less impact on the healing effect of the microcapsule in cementitious composite.

3. Epoxy resin microcapsule could be kept in good shape after stirring and forming in cement paste, and combined with cement paste closely, which is the precondition for playing the healing role. Epoxy resin microcapsule had better dispersion in cement paste section, which is easier to play its healing function. Epoxy resin microcapsule also could rupture in cement paste, and the main fracture behavior of the microcapsule was based on small hole ruptured when cement paste did not occur macro damage.

\section{Acknowledgments}

This project was financially supported by the National Natural Science Foundation of China (No.51972158 and No.52062032) and Major Discipline Academic and Technical Leaders Training Program of Jiangxi Province (No.20204BCJ22001).

\section{REFERENCES}

1. White, S.R., Sottos, N.R., Geubelle, P.H., Moore, J.S., Kessler, M.R., $\quad$ Sriram, S.R., $\quad$ Brown, E.N., Viswanathan, S. Autonomic Healing of Polymer Composites Nature 409 (6822) 2001: pp. 794-797. https://doi.org/10.1038/35057232

2. Li, W.T., Dong, B.Q., Yang, Z.X., Xu, J., Chen, Q., Li, H.X., Xing, F., Jiang, Z.W. Recent Advances in Intrinsic Self-healing Cementitious Materials Advanced Materials 30 (17) 2018: pp. 1705679. https://doi.org/10.1002/adma.201705679.

3. Wang, J.Y., Soens, H., $\quad$ Verstraete, W. Self-healing Concrete by Use of Microencapsulated Bacterial Spores Cement and Concrete Research $56(2)$ 2014: pp. $139-152$. https://doi.org/10.1016/j.cemconres.2013.11.009

4. Han, N.X., Xing, F. A Comprehensive Review of the Study and Development of Microcapsule Based Self-resilience Systems for Concrete Structures at Shenzhen University Materials $10(1)$ 2017: pp. 2-26. https://doi.org/10.3390/ma10010002

5. Lv, L.Y., Schlangen, E., Yang, Z.X., $\quad$ Xing, F. Micromechanical Properties of a New Polymeric Microcapsule for Self-healing Cementitious Materials Materials 9 (12) 2016: pp. 1025-1040. https://doi.org/10.3390/ma9121025

6. Yang, Z.X., Hollar, J., He, X.D., Shi, X.M. A Self-healing Cementitious Composite Using Oil Core/silica Gel Shell Microcapsules Cement and Concrete Composite 33 (4) 2011: pp. 506-512. https://doi.org/10.1016/j.cemconcomp.2011.01.010

7. Hu, T., Yao, X., Zhu, H.J. The preparation and Self-healing Performance of Poly (Urea-formaldehyde) Microcapsules for Set Cement Polymer Materials Science and Engineering 28 (8) 2012: pp. 148 -151(in Chinese). https://doi.org/ 10.16865/j.cnki.1000-7555.2012.08.037

8. Dong, B.Q., Han, N.H., Zhang, M., Wang, X.F., Cui, H.Z., Xing, F. A Microcapsule Technology Based Self-healing System for Concrete Structures Journal of Earthquake and Tsunami 7 (3) 2013: pp. 1350014. 
https://doi.org/10.1142/s1793431113500140

9. Li, W.T., Zhu, X.J., Zhao, N., Jiang, Z.W. Preparation and Properties of Melamine Urea-formaldehyde Microcapsules for Self-healing of Cementitious Materials Materials 9 (3) 2016: pp. $152-168$. https://doi.org/10.3390/ma9030152

10. Dong, B.Q., $\quad$ Fang, G.H., Ding, W.J., Liu, Y.Q., Zhang, J.C., Han, N.X., Xing, F. Self-healing Features in Cementitious Material with Urea-formaldehyde/Epoxy Microcapsules Construction and Building Materials 106 2016: pp. $608-617$.

https://doi.org/10.1016/j.conbuildmat.2015.12.140

11. Wang, X.F., Xing, F., Zhang, M., Han, N.X., Qian, Z.W. Experimental Study on Cementitious Composites Embedded with Organic Microcapsules Materials 6(9) 2013: pp. $4064-4081$. https://doi.org/10.3390/ma6094064

12. Wang, X.F., Sun, P.P., Han, N.X., Xing, F. Experimental Study on Mechanical Properties and Porosity of Organic Microcapsules Based Self-healing Cementitious Composite Materials 10 (1) 2017: pp. 20-37. https://doi.org/10.3390/ma10010020

13. Ollier, R.P., Penoff, M.E., Alvarez, V.A. Microencapsulation of Epoxy Resins: Optimization of Synthesis Conditions Colloids and Surfaces A:
Physicochemical and Engineering Aspects 2016: pp. 27-38. https://doi.org/10.1016/j.colsurfa.2016.09.081

14. Van, T.K., De, B.N. Self-Healing in Cementitious Materials-A Review $\quad$ Materials 6 (6) 2013: pp. 2182-2217. https://doi.org/ 10.3390/ma6062182

15. Ghosh, S.K. Self-healing Materials: Fundamentals, Design Strategies and Applications, Wiley-VCH, 2009. https://doi.org/10.1016/S1369-7021(09)70143-9

16. Dong, K.M., Cho, Y.J., Choi, J.Y., Kim, B.J., Jin, S.W., Chung, C.M. Low-temperature Self-healing of A Microcapsule-type Protective coating Materials $10(9)$ 2017: pp. $1079-1092$. https://doi.org/10.3390/ma10091079

17. Shen, X.J., Liu, K.H., Gao, D.Y., Sun, T., Yang, L., Wu, Z.J. Research Progress in Microencapsulated Selfhealing Polymer Matrix Composite Acta Materiae Compositae Sinic 9 2018: pp. 2303-2320 (in Chinese). https://doi.org/10.13801/j.cnki.fhclxb.20180111.00

18. Patrick, J.F., Robb, M.J., Sottos, N.R., Moore, J.S., White, S.R. Polymers with Autonomous Life-cycle Control Nature 540 (7633) 2016: pp. 363-370. https://doi.org/10.1038/nature21002 